\title{
An Energy-Based Centrality for Electrical Networks
}

\author{
Ruiyuan Kong ${ }^{1}$, Congying $\mathrm{Han}^{2}$, Tiande Guo ${ }^{1}$, Wei Pei ${ }^{3}$ \\ ${ }^{1}$ School of Mathematical Sciences, University of Chinese Academy of Sciences, Beijing, China \\ ${ }^{2}$ College of Humanities and Social Sciences, University of Chinese Academy of Sciences, Beijing, China \\ ${ }^{3}$ Institute of Electrical Engineering, Chinese Academy of Sciences, Beijing, China \\ Email: xyzkong@126.com
}

Received December, 2012

\begin{abstract}
We present an energy-based method to estimate centrality in electrical networks. Here the energy between a pair of vertices denotes by the effective resistance between them. If there is only one generation and one load, then the centrality of an edge in our method is the difference between the energy of network after deleting the edge and that of the original network. Compared with the local current-flow betweenness on the IEEE 14-bus system, we have an interesting discovery that our proposed centrality is closely related to it in the sense of that the significance of edges under the two measures are very similar.
\end{abstract}

Keywords: Centrality; Energy; Effective Resistance; Current-flow Betweenness

\section{Introduction}

The electrical network is one of the most critical and complex infrastructure networks in modern society. There are some important issues which are keys to the performance of the network. Reliable electric power supply, for example, is crucial for many devices and its disturbances may disrupt the devices or even paralyze the network. This brings the concern about reliability and resilience to disturbances and failures of various types of infrastructure systems, and a corresponding demand for methods of analyzing the vulnerabilities of the electrical network [1]. Moreover, the blackouts of the North American and Italian electric power grids in 2003 exposed the weaknesses of the electrical network. The weakness and vulnerable analysis about the electrical network have been widely studied in the past years [1-4].

With recent advances in network and graph theory, many researchers have applied centrality measures to complex networks in order to study network properties. Various centrality measures have been defined. They draw links between the structure of networks and the vulnerability to certain types of failures, and are used to identify the most vulnerable elements of a network. Traditionally, there are four centrality measures within network analysis, i.e., degree centrality, betweenness, closeness, and eigenvector centrality. The degree metric utilizes the local information. Closeness and betweenness utilize the shortest path information. And the eigenvector metric rely on the Laplacian matrix of the group. All of them consider only the topological properties but not the actual physical flow through the power system. Moreover, the betweenness and closeness centrality postulate that the information or flow transfer along the shortest path, but this is not true for the current in the electrical network. A series of centrality measures considering the physical flow are proposed. [5] proposed a so-called random-walk betweenness, counting how often a node is traversed by a random walk between two other nodes. This centrality is known to be useful for finding vertices of high centrality that do not lie on the shortest path. Actually, the random-walk betweenness is closely related to the current-flow betweenness proposed in [6]. The paper derives the metric straightforward from the electrical current and proves that the current-flow closeness is in fact identical with the information centrality [7]. Some papers proposed their measures which are actually of no difference with the current-flow betweenness though they didn't point that directly. For example, [8] proposed an electrical centrality measure based on the impedance matrix which is similar to the current-flow centrality. Besides, they pointed out the differences of the topology of power grids from that of Erdos-Renyi random graphs, the "small-world" networks or "scale-free" networks but the power networks appear to have a scale-free network structure under their proposed measure. However, as the indication of [9], the proposed electrical centrality measure in [8] was defined incorrectly. But a simple analysis shows that the revised measure was the right currentflow betweenness.

The betweenness above needs to take into account all pairs of nodes in the networks. [10] considered only the 
pairs of generations and load nodes. Besides, they considered some other features of power systems such as power transfer distribution and line flow limits, and got that according to the un-served energy after the network being attacked the nodes ranked highly is more vulnerable.

The centralities defined before are not so easy to understand. This paper will propose an easy-understanding method which is based on the effective resistance. The effective resistance between a pair of vertices $s$ and $t$ is the potential difference between them ensuring a current of size 1 from $s$ to $t$, and can be seen as the total energy in the system. The effective resistance is local in some sense. Its global form is the Kirchhoff index, which is based on the resistance-distance matrix introduced in 1993 by Klein and Randic and defined as the effective resistance between pairs of vertices [11]. The Kirchhoff index is often used to quantify the structural attributes of the graph. See [12,13] for more information.

This paper is organized as follows. In Section 2, we introduce some preliminary concepts about centrality measures, the effective resistance and so on. In section 3 , the definition of our measure based on energy and the variation of current-flow betweenness, that is, the local current-flow betweenness is given. Section 4 provides the comparisons with the current-flow betweenness centrality and other centrality measures. Conclusions are drawn in Section 5.

\section{Preliminaries}

\subsection{Betweenness}

Vertex betweenness, first introduced by Freeman in 1977 [14], is one of the most used centrality measure. It reflects the occurrence degree of a node on the shortest path between any pair of nodes. Given a undirected graph $G(V, E)$, where $V$ is the set of vertices and $E$ is the set of edges, the betweenness of a node $v$ is defined by:

$$
C_{b}(v)=\frac{\sum_{s \neq v \neq t \in V} \sigma_{s t}(v) / \sigma_{s t}}{(n-1)(n-2) / 2},
$$

where $\sigma_{s t}$ and $\sigma_{s t}(v)$ are the number of shortest paths from $s$ to $t$ and the number of shortest paths from $s$ to $t$ through $v$. Girvan and Newman [15] generalized the vertex betweenness to edges and proposed edge betweenness which is defined as the number of shortest paths between pairs of vertices that run along it and used to find which edges are most important. If there is more than one shortest path between a pair of vertices, then take them as one path. The edge betweenness is given by:

$$
C_{b}(v)=\frac{\sum_{s \neq t \in V} \sigma_{s t}(e) / \sigma_{s t}}{n(n-1) / 2} .
$$

It is found that the removal of the nodes or edges with large betweenness will put the network at high risk to be disconnected.

\subsection{Current-Flow Betweenness}

The current-flow betweenness here is based on the definition of [6]. An electrical network is a graph $N=$ $(V, E, c)=(G, c)$, together with a function $c: \rightarrow R^{+}$, where $c_{e}=c(\mathrm{e})$ is the reciprocal of the resistance of the edge $e$. Given a supply of size 1 from a source $s$ to a sink $t$, the throughput of an edge $e$ and an inner vertex $v$ is defined by:

$$
\begin{aligned}
& \tau_{s t}(e)=|w(\vec{e})|, \\
& \tau_{s t}(v)=\frac{1}{2} \sum_{e: v \in e}|w(\vec{e})| .
\end{aligned}
$$

Define the edge and vertex current-flow betweenness respectively by:

$$
\begin{aligned}
& c_{C B}(e)=\frac{1}{n(n-1)} \sum_{s, t \in V} \tau_{s t}(e), \\
& C_{C B}(v)=\frac{1}{(n-1)(n-2)} \sum_{s, t \in V} \tau_{s t}(v) .
\end{aligned}
$$

The current-flow betweenness is reasonable for that the current is unique by Lemma 1 of [6].

Besides, Brandes proposed also the current-flow closeness centrality which is a variation of closeness centrality. It is defined by:

$$
c_{C C}(s)=\frac{n_{C}}{\sum_{t \neq s} p_{s t}(s)-p_{s t}(t)}
$$

for all $s$ in $V$, where $p(s)$ refers to the voltage in the vertex s. Moreover, Brandes proved that the currentflow closeness centrality equals information centrality.

\subsection{Effective Resistances, Energy and Kirchhoff Index}

Now we give the definition of effective resistances. The effective resistance is the potential difference between $s$ and $t$ ensuring a current of size 1 from $s$ to $t$ and denoted by $R_{s t}(G)$.

The total energy in a network is defined as:

$$
\sum_{x y \in E} w_{x y}^{2}=\sum_{x y \in E}\left(V_{x}-V_{y}\right)^{2} c_{x y}=\sum_{x y \in E}\left(V_{x}-V_{y}\right) w_{x y}
$$

where $V_{x}$ is absolute potential and $w_{x y}=\left(V_{x}-V_{y}\right) c_{x y}$ is the energy in the edge between $x$ and $y$. By Lemma 2 of [6], there are unique potentials. So the definition of the total energy is reasonable.

Kirchhoff index is the sum of the effective resistances over all pairs of vertices:

$$
K f(G)=\sum_{i<j} r_{i j},
$$

where $r_{i j}$ is the effective resistance between $i$ and $j$. It is 
proved that Kirchhoff index satisfies

$$
K f(G)=\sum_{i=1}^{n-1} \frac{1}{\lambda_{i}},
$$

where $\left\{\lambda_{i}, i=1, \ldots, n-1\right\}$ are the nonzero eigenvalues of the Laplacian matrix of $G$.

\section{Centrality Metric Based on Energy}

It is known that the total energy in an electric current with size 1 from $s$ to $t$ is the effective resistance [16]. If there is only one source $s$ and one sink $t$, then the change in the energy is the change in the effective resistance between $s$ and $t$. Therefore to measure the influence of removing one edge we can define a 'metric' based on the variant of energy by:

$$
\Delta E(e)=R_{s t}(G \backslash e)-R_{s t}(G),
$$

where $G \backslash e$ is the graph deleted by the edge $e$. Though $\Delta E(e)$ is not a strict definition of a metric, we regard it as a metric since we only focus on the results under the metric but not their values. The larger $\Delta E(e)$, the greater the risk for the network to be damaged when deleting the edge $e$. If there is no connection between $s$ and $t$ after deleting the edge $e$, then $\Delta E(e)=\infty$. In other words, the edge $e$ with $\Delta E(e)=\infty$ is very important to the network. By the monotonicity principle, Corollary 7 of [16], $\Delta E(e)$ is nonnegative for all edges.

For the network with sources $S$ and sinks $T$ where $S \cap T=\varnothing$, the metric based on energy is defined by

$$
\Delta E(e)=\frac{\sum_{s \in S, t \in T} R_{s t}(G \backslash e)-R_{s t}(G)}{|S||T|},
$$

where $|S|$ denotes the cardinality of the set $S$. The energy-based centrality can be seen as a local metric to measure the importance of an edge. Analogous to the definition of betweenness, we consider the whole importance of an edge, that is,

$$
\Delta E^{\prime}(e)=\frac{\sum_{s \neq t} R_{s t}(G \backslash e)-R_{s t}(G)}{n(n-1) / 2} .
$$

If we define

$$
\Delta K f(e)=\frac{K f(G \backslash e)-K f(G)}{n(n-1) / 2},
$$

then it is easy to check that $\Delta K f(e)=\Delta E^{\prime}(e)$ by the definition of the Kirchhoff index. So we call the case defined by Equation (1) the edge Kirchhoff-based centrality. Analogous to the definition of vertex current-flow betweenness, we can also define the vertex energy-based centrality and vertex Kirchhoff-based centrality by

$$
\Delta E_{v}(u)=\frac{\sum_{e \in \Gamma(u)} \Delta E(e)}{|\Gamma(u)|},
$$

$$
\Delta K f_{v}(u)=\frac{\sum_{e \in \Gamma(u)} \Delta K f(e)}{|\Gamma(u)|},
$$

where $\Gamma(u)$ denotes by the adjacent edges of the vertex $u$.

Next we take Theorem 1 of [17] as a lemma which will be used to compute the energy-based centrality.

Lemma 1. Let $G$ be a connected graph on $n \geq 3$ vertices and $1 \leq i \neq j \leq n$. Let $L(i)$ be the submatrix obtained from the Laplacian matrix $L$ of the graph $G$ by deleting its ith row and ith column and $L(i, j)$ be the submatrix obtained from the Laplacian matrix $L$ by deleting its ith and jth rows and the ith and jth columns. Then the effective resistance $r_{i j}$ between $i$ and $j$ satisfies

$$
r_{i j}=\frac{\operatorname{det}(L(i, j))}{\operatorname{det}(L(i))} .
$$

Note that the graph $G$ in the lemma above can be seen as a graph with one unit resistance on each edge of the network. Following the steps of its proof, we can easily check that the result holds for the graph $G$ with different resistances on the edges and the Laplacian matrix $L$ being replaced by the admittance matrix of the network. Though in general the complexity of computing the effective resistance using equation (2) is $O\left(n^{3}\right)$, the remarkably simple expression is still very valuable.

Recall the argument of the reference [10] in the introduction. The authors utilized a local idea considering the electrical betweeness only between the pairs of generations and loads with some other restriction, but they didn't point out that clearly and didn't give the corresponding simulation results. Thus this paper gives the definition and simulation clearly, and calls it the local current-flow betweenness. Analogous to the current-flow betweenness, for the network with sources $S$ and sinks $T$ we define the edge local current-flow betweenness by

$$
L C_{b}(e)=\frac{\sum_{s \in S, t \in T} \sigma_{s t}(e)}{|S \| T|} .
$$

The vertex local current-flow betweenness is defined similarly and denoted by $L C_{v}(u)$.

\section{Numerical Analysis}

In this section, the edge (vertex) energy-based metric and the edge (vertex) Kirchhoff-based metric are compared with the edge (vertex) current-flow betweenness, the edge (vertex) local current-flow betweenness and the closeness centrality on the IEEE 14-bus. The IEEE 14bus consists of 20 lines and 14 buses including 2 generators and 2 loads, as shown in Figure 1. And Figure 2 is the graph representation of IEEE 14-bus transmission network. The circles with labeled $G$ represent the generator nodes, the circles with labeled $L$ represent the load 


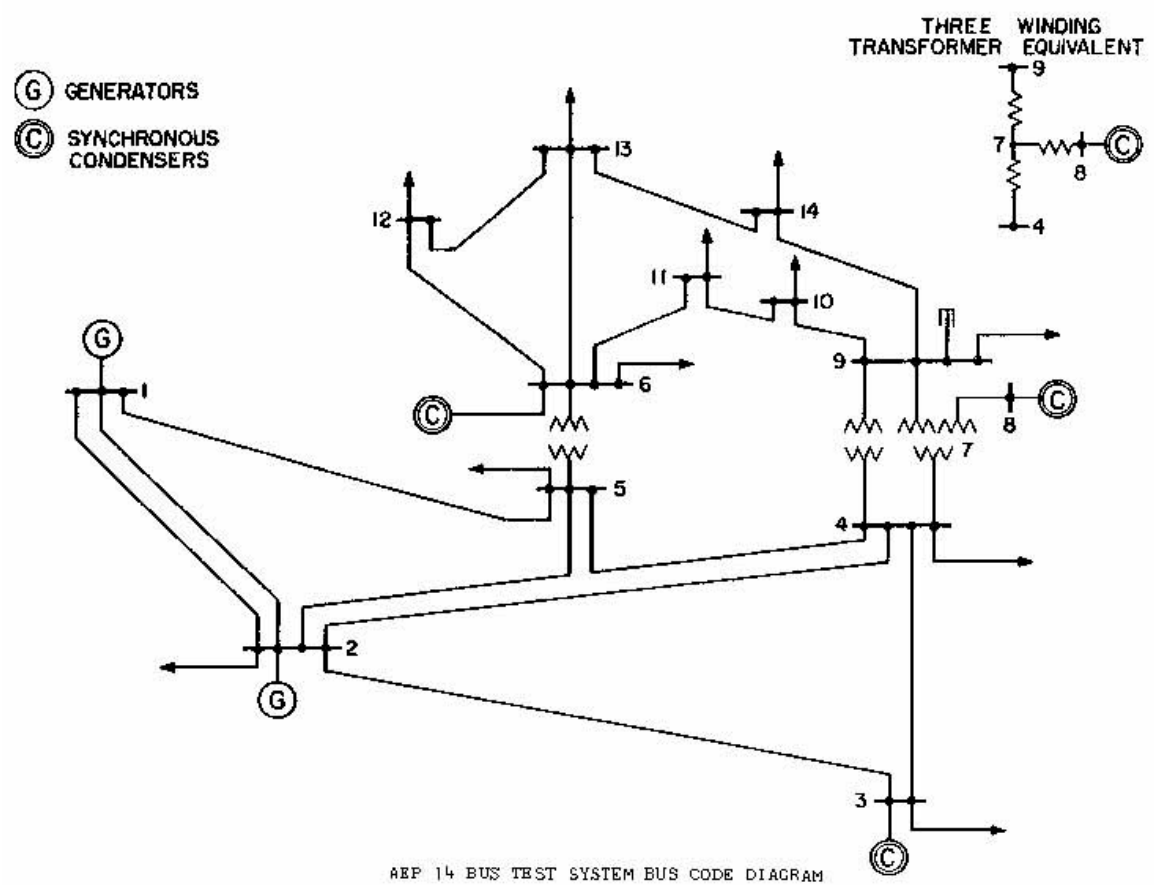

Figure 1. Transmission network IEEE 14-bus.

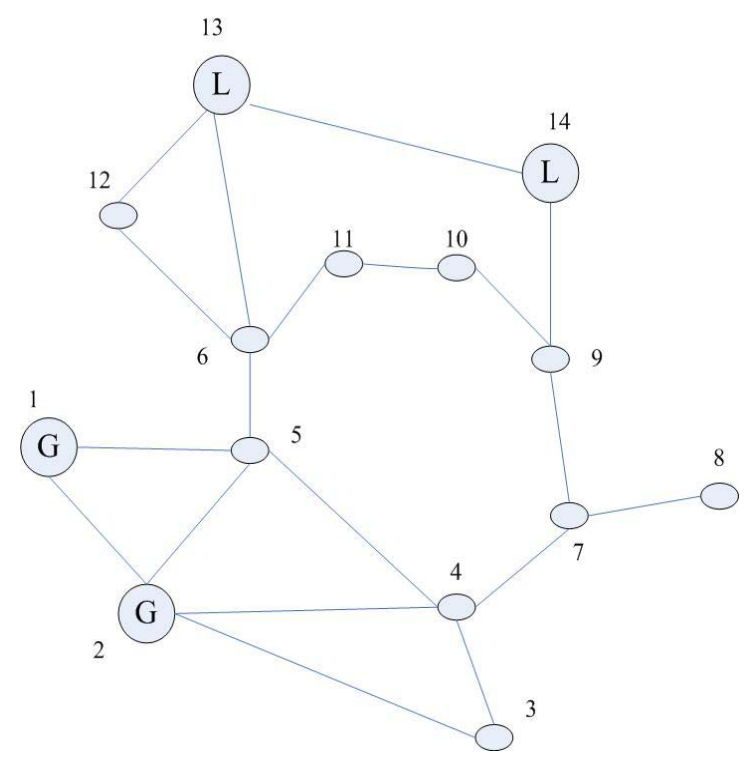

Figure 2. The graph representation of IEEE 14-bus transmission network.

nodes and the ellipses represent the transmission nodes. It is known that for the high-voltage transmission network in a power grid the reactance is usually the dominant component of a line impedance. Thus for the purpose of simplicity, we take the reactance as the edge weights. And the bus 8 will not affect the effective resistance between the generations and loads, thus we don't consider it. To keep in accord, we compute other centrality metrics based on the case above. Besides, the bus 8 is of low betweenness centrality by [18].
Table 1 ranks the edges according to the four edge centralities. The edge current-flow betweenness, edge local current-flow betweenness, edge energy-based measure and edge Kirchhoff-based measure are abbreviated as B, Local-B, E-based and Kf-based respectively. All the four methods rank the edge 5-6 first, which says that the edge 5-6 is very possible to be the most important branch in this network. It shows that the edge betweenness and the edge Kirchhoff-based centrality are quite different with each other and with the other two measures. 
Table 1. The importance order of edges from high to low.

\begin{tabular}{ccccc}
\hline Order & B & Local-B & E-based & Kf-based \\
\hline 1 & $5-6$ & $5-6$ & $5-6$ & $5-6$ \\
2 & $1-2$ & $6-13$ & $9-14$ & $9-10$ \\
3 & $7-9$ & $9-14$ & $13-14$ & $9-14$ \\
4 & $9-10$ & $1-2$ & $6-13$ & $7-9$ \\
5 & $4-7$ & $13-14$ & $1-2$ & $6-11$ \\
6 & $4-5$ & $7-9$ & $4-7$ & $10-11$ \\
7 & $6-11$ & $4-7$ & $7-9$ & $4-7$ \\
8 & $10-11$ & $2-4$ & $1-5$ & $13-14$ \\
9 & $9-14$ & $2-5$ & $4-9$ & $6-13$ \\
10 & $6-13$ & $1-5$ & $2-4$ & $1-2$ \\
11 & $13-14$ & $4-9$ & $2-5$ & $3-4$ \\
12 & $1-5$ & $3-4$ & $6-12$ & $12-13$ \\
13 & $2-4$ & $2-3$ & $12-13$ & $6-12$ \\
14 & $2-5$ & $12-13$ & $9-10$ & $4-5$ \\
15 & $2-3$ & $6-12$ & $10-11$ & $4-9$ \\
16 & $3-4$ & $9-10$ & $6-11$ & $2-3$ \\
17 & $12-13$ & $10-11$ & $2-3$ & $1-5$ \\
18 & $6-12$ & $6-11$ & $3-4$ & $2-4$ \\
19 & $4-9$ & $4-5$ & $4-5$ & $2-5$ \\
\hline 19 & & & & \\
\hline
\end{tabular}

Table 2. The importance order of nodes from high to low.

\begin{tabular}{cccccc}
\hline Order & V-B & VLocal-B & C & VE-based & VKf-based \\
\hline 1 & 4 & 2 & 12 & 14 & 10 \\
2 & 5 & 13 & 14 & 6 & 9 \\
3 & 6 & 14 & 3 & 9 & 7 \\
4 & 9 & 1 & 11 & 13 & 14 \\
5 & 2 & 6 & 13 & 5 & 11 \\
6 & 7 & 5 & 1 & 7 & 6 \\
7 & 1 & 4 & 10 & 1 & 5 \\
8 & 13 & 9 & 7 & 2 & 13 \\
9 & 10 & 7 & 2 & 4 & 12 \\
10 & 11 & 3 & 6 & 12 & 4 \\
11 & 14 & 12 & 9 & 10 & 3 \\
12 & 3 & 10 & 5 & 11 & 1 \\
13 & 12 & 11 & 4 & 3 & 2 \\
\hline
\end{tabular}

But there is a strong correlation between the edge local current-flow betweenness and our edge energy-based centrality. The first 5 important edges are the same and their orders are of little difference. Moreover, the edges ranking from 5 to 11 are also consistent with their orders being little different. In fact, for any edge $e$ in the first 11 edges, the difference between the ranking of $e$ in the two cases is at most 2. However, the complexity of computing the edge energy-based centrality is lower than that of computing the edge local current-flow betweenness. And the complexities for computing the two centrality are both $O\left(n^{3}\right)$. But the latter has a much more clear expression. In practice, we can use both to measure the importance of two edges from different perspectives. If we give the edge ranking first the score 19, the edge ranking second the score $18, \ldots$, the edge ranking last the score 1 , and denote $i-j$ by the edge between the ver- tex $i$ and $j$. Then given the ordered sequence $\{1-2,1-5$, 2-3, 2-4, 2-5, 3-4, 4-5, 4-7, 4-9, 5-6, 6-11, 6-12, 6-13, 7-9, $9-10,9-14,10-11,12-13,13-14\}$, denote by $X_{1}$ and $X_{2}$ the ranking lists for energy-based centrality and edge local current-flow betweenness respectively. Using linear regression to check whether $X_{1}$ and $X_{2}$ are relevant, we get that the adjusted $R^{2}$ equals 0.814 and the p-value is very close to 0 . This shows that they are strongly correlated.

\section{Conclusions}

This paper considers measures of centrality that are used to rank the importance of the nodes or edges in an electrical network. New methods of centrality are defined from the perspective of the energy of a network. More specifically, we use the variant of the effective resistances between the generations and loads after deleting an edge or a node to measure its importance, similar to which we also define a Kirchhoff-based measure with the effective resistances being replaced the Kirchhoff index. Besides, we propose the local current-flow betweenness in the most simple way more clearly.

Based on defined measures, experiments are performed on IEEE 14-bus and some interesting results are discovered. It has been found that our proposed edge energybased measure is very similar to the local current-flow betweenness, in the sense that the importance rankings of the edges in the two measures are of little difference. While the expression of computing the energy-based measure is very simple and clear. Besides, from the experiments we get that the current-flow betweenness is very different from the local current-flow. However, it is difficult to judge which are more accurate. Moreover, we verify that the current-flow betweenness is closely related to the closeness centrality in our experiments. However, more tests and analysis need to be done in order to validate the proposed measure, to find the most effective measure and to dig deep to see which nodes or edges are the real most important nodes or edges.

\section{Acknowledgements}

This work is supported by CAS Knowledge Innovation Program (grant number KGCX2-RW-329) and National Natural Science Foundation of P.R. China (grant number 10831006, 71271204).

\section{REFERENCES}

[1] R. Albert, I. Albert and G. L. Nakarado, "Structural Vulnerability of the North American Power Grid,” Physical Review E, Vol. 69, No. 2, 2004, pp. 1-4. doi:10.1103/PhysRevE.69.025103

[2] B. A. Carreras, V. E. Lynch, I. Dobson and D. E. New- 
man, "Critical Points and Transitions in an Electric Power Transmission Model for Cascading Failure Blackouts," Chaos, Vol. 12, No. 4, 2002, pp. 985-994.

doi: 10.1063/1.1505810

[3] P. Crucittia, V. Latorab and M. Marchioric, "A Topological Analysis of the Italian Electric Power Grid," Physica A: Statistical Mechanics and its Applications, Vol. 338, No. 1-2, 2004, pp. 92-97. doi:10.1016/j.bbr.2011.03.031

[4] M. Rosas-Casals, S. Valverde and R. V. Solé, “Topological Vulnerability of the European Power Grid Under Errors and Attacks," International Journal of Bifurcation and Chaos in Applied Sciences and Engineering, Vol. 17, No. 7, 2007, pp. 2465-2475. doi: $10.1142 / \mathrm{S} 0218127407018531$

[5] M. E. J. Newman, "A Measure of Betweenness Centrality Based on Random Walks,” Social Networks, Vol. 27, No. 1, 2005, pp. 39-54. doi:10.1016/j.socnet.2004.11.009

[6] U. Brandes and D. Fleischer, "Centrality Measures Based on Current Flow," Proceedings of the 22nd Symposium on Theoretical Aspects of Computer Science, Lecture Notes in Computer Science, Springer, Berlin, Vol. 3404, 2005, pp. 533-544. doi:10.1007/978-3-540-31856-9 44

[7] K. A. Stephenson and M. Zelen, "Rethinking Centrality: Methods and Examples," Social Networks, Vol. 11, No. 1, 1989, pp. 1-37. doi:10.1016/0378-8733(89)90016-6

[8] P. Hines and S. Blumsack, "A Centrality Measure for Electrical Networks," Proceedings of the 41st Hawaii International Conference on System Sciences, Hawaii, 7-10 January 2008, p.185. doi:10.1109/HICSS.2008.5

[9] Z. H. Wang, A. Scaglione and R. J. Thomas, "Electrical Centrality Measures for Power Grids," Control and Optimization Methods for Electric Smart Grids, Power Electronics and Power System, Springer, New York, Vol.3, 2012, pp. 239-255. doi:10.1007/978-1-4614-1605-0_12

[10] E. Bompard, D. Wu and F. Xue, "The Concept of Betweenness in the Analysis of Power Grid Vulnerability," Complexity in Engineering, Rome, 22-24 February, 2010, pp. 52-54. doi:10.1109/COMPENG.2010.10

[11] D. J. Klein and M. Randic, "Resistance Distance," Journal of Mathematical Chemistry, Vol. 12, No. 1, 1993, pp.81-95. doi:10.1007/BF01164627

[12] K. C. Das, A. D. Gungor and A. S. Cevik, " On Kirchhoff Index and Resistance-Distance Energy of a Graph," MATCH Communications in Mathematical and in Computer Chemistry, Vol. 67, No. 2, 2012, pp. 541-556.

[13] B. Zhou and N. Trinajstic, "On Resistance-Distance and Kirchhoff Index,” Journal of Mathematical Chemistry, Vol. 46, No. 1, 2009, pp. 283-289. doi:10.1007/s10910-008-9459-3

[14] L. C. Freeman , "A Set of Measures of Centrality Based On Betweenness,” Sociometry, Vol. 40, No. 1, 1977, pp. 35-41.

[15] M. Girvan and M. E. J. Newman, "Community Structure in Social and Biological Networks," Proceedings of the National Academy of Sciences, Vol. 99, No. 12, 2002, pp. 7821-7826. doi:10.1073/pnas.122653799

[16] B. Bollobás, “Modern Graph Theory,”3rd Edition, Springer-Verlag, New York, 2003.

[17] R. Bapat, I. Gutman and W. Xiao, "A Simple Method for Computing Resistance Distance," Zeitschrift für Naturforschung, Vol. 58a, No. 9-10, 2003, pp. 494-498.

[18] E. Zio , R. Piccinelli and M. Delfanti , “Application of the Load Flow and Random Flow Models for the Analysis of Power Transmission Networks," Reliability Engineering and System Safety, Vol. 103, 2012, pp. 102-109. doi:10.1016/j.ress.2012.02.005 